Preface

\title{
Editorial Compilation IX
}

\author{
Emmanuel J. Favaloro, PhD, FFSc (RCPA) ${ }^{1}$ Giuseppe Lippi, MD ${ }^{2}$ \\ ${ }^{1}$ Department of Haematology, Institute of Clinical Pathology and \\ Medical Research (ICPMR), Sydney Centres for Thrombosis and \\ Haemostasis, Westmead Hospital, Westmead, Australia \\ 2 Section of Clinical Biochemistry, University of Verona, Verona, Italy \\ Semin Thromb Hemost 2021;47:6-10.
}

Welcome to the latest issue of Seminars in Thrombosis and Hemostasis (STH) published under the "banner" of "Editorial Compilation," this being the ninth such issue. Although Seminars in Thrombosis and Hemostasis is historically a themedriven publication, ongoing opportunities arise to publish issues containing wide-ranging contributions of current interest and controversy, and which do not quite match a current themed issue in progress. We also require a medium to enable publication of accepted unsolicited manuscripts as well as contributions from our Eberhard F. Mammen Young Investigator Award winners (-Table 1). As is now standard for this compilation series, the current issue has a mixture of content that comprises the above elements, as well as broadly fitting within the standard themes of "thrombosis" and "bleeding."

This issue begins fittingly with a contribution from one of the recent Eberhard F. Mammen Young Investigator Award winners, Elisa Danese, ${ }^{1}$ and colleagues, on the role of epigenetics in regulation of hemostatic balance. ${ }^{2}$ Epigenetics, as a term, is conventionally used to explain the intricate interplay between genes and environment, and is now regarded as a fundamental of developmental biology. Epigenetic alterations, mostly encompassing DNA methylation, histone tail modifications, and generation of microRNAs, play an important, though still incompletely explored, role in both primary and secondary hemostasis. Epigenetic variations may interplay with platelet function and their responsiveness to antiplatelet drugs, and they may also exert a substantial contribution in modulating production and release into the bloodstream of proteins involved in blood coagulation and fibrinolysis. The emerging evidence outlined in this review may have substantial biological and clinical implications. An enhanced understanding of posttranscriptional mechanisms would help to clarify some remaining enigmatic issues in primary and secondary hemostasis, which cannot be thoughtfully explained by genetics or biochemistry alone. Increased understanding would also pave the way to developing innovative tests for better assessment of

Address for correspondence Emmanuel J. Favaloro, PhD, FFSc (RCPA), Department of Pathology and Medical Research (ICPMR), Westmead Hospital, Westmead, 2145, Australia (e-mail: emmanuel.favaloro @health.nsw.gov.au). individual risk of bleeding or thrombosis. The accurate recognition of key epigenetic mechanisms in hemostasis would then contribute to identify new biological pathways and putative therapeutic targets, thus allowing to develop innovative agents that could be helpful for preventing or managing a vast array of hemostasis disturbances.

Next is a contribution around the general topic of coagulation and "clotting triggers" from He and colleagues. ${ }^{3}$ Blood coagulation comprises a series of enzymatic reactions leading to thrombin generation and fibrin formation. This process is commonly illustrated in a waterfall-like manner, referred to as the coagulation cascade. In vivo, this "cascade" is initiated through the tissue factor (TF) pathway once subendothelial TF is exposed and bound to coagulation factor VII (FVII) in blood. In vitro, a diminutive concentration of recombinant TF (rTF) is used as clotting trigger in various global hemostasis assays such as the calibrated automated thrombogram, or methods that assess fibrin turbidity and fibrin viscoelasticity tests such as rotational thromboelastometry. These assays aim to mimic in vivo global coagulation and are useful in assessing hyper-/hypocoagulable disorders or monitoring therapies with hemostatic agents. An excess of rTF, a sufficient amount of negatively charged surfaces, various concentrations of exogenous thrombin, recombinant activated FVII, or recombinant activated FIXa are also used to initiate the activation of specific subprocesses of the coagulation cascade in vitro. These approaches offer important information on certain specific coagulation pathways, while alterations in pro-/anticoagulants not participating in these pathways remain undetectable by these methods. Reviewing available data, the authors seek to enhance our knowledge of how the choice of clotting trigger affects the outcome of hemostasis assays and address the call for further investigations on this topic.

This issue of STH continues with the exploration of hemostasis from a contribution from Dr. Amin Alamin, ${ }^{4}$ on
(C) 2021. Thieme. All rights reserved.

Thieme Medical Publishers, Inc., 333 Seventh Avenue, 18th Floor, New York, NY 10001, USA
DOI https://doi.org/ 10.1055/s-0040-1721751. ISSN 0094-6176.
Compilation IX; Guest Editors: Emmanuel J. Favaloro, PhD, FFSc (RCPA) and Giuseppe Lippi, MD. 
Table 1 Past STH editorials related to Eberhard F. Mammen Award Announcements

\begin{tabular}{|l|l|}
\hline 1 & Favaloro EJ. 2011 Eberhard F. Mammen Award Announcements. Semin Thromb Hemost 2011;37(5):431-439 \\
\hline 2 & Favaloro EJ. 2012 Eberhard F. Mammen Award Announcements. Semin Thromb Hemost 2012;38(5):425-432 \\
\hline 3 & Favaloro EJ. 2013 Eberhard F. Mammen Award Announcements. Semin Thromb Hemost 2013;39(6):567-574 \\
\hline 4 & $\begin{array}{l}\text { Favaloro EJ. 2014 Eberhard F. Mammen Award Announcements: Part II-Young Investigator Awards. } \\
\text { Semin Thromb Hemost 2014;40(7):718-723 }\end{array}$ \\
\hline 5 & $\begin{array}{l}\text { Favaloro EJ. 2015 Eberhard F. Mammen Award Announcements: Part II-Young Investigator Awards. } \\
\text { Semin Thromb Hemost 2015;41(8):809-815 }\end{array}$ \\
\hline 6 & $\begin{array}{l}\text { Favaloro EJ. 2016 Eberhard F. Mammen Award Announcements: Part II-Young Investigator Awards. } \\
\text { Semin Thromb Hemost 2017;43(3):235-241 }\end{array}$ \\
\hline 7 & $\begin{array}{l}\text { Favaloro EJ. 2017 Eberhard F. Mammen Award Announcements: Part II-Young Investigator Awards. } \\
\text { Semin Thromb Hemost 2018;44(2):81-88 }\end{array}$ \\
\hline 8 & $\begin{array}{l}\text { Favaloro EJ. 2018 Eberhard F. Mammen Award Announcements: Part II-Young Investigator Awards. } \\
\text { Semin Thromb Hemost 2019;45(2):123-129 }\end{array}$ \\
\hline 9 & $\begin{array}{l}\text { Favaloro EJ. 2019 Eberhard F. Mammen Award Announcements: Part I-Most Popular Articles. } \\
\text { Semin Thromb Hemost 2019;45(3):215-224 }\end{array}$ \\
\hline 10 & $\begin{array}{l}\text { Favaloro EJ. 2019 Eberhard F. Mammen Award Announcements: Part II-Young Investigator Awards. } \\
\text { Semin Thromb Hemost 2020;46(2):105-113 }\end{array}$ \\
\hline 11 & $\begin{array}{l}\text { Favaloro EJ. 2020 Eberhard F. Mammen Award Announcements: Part I-most popular articles. } \\
\text { Semin Thromb Hemost 2020;46(4):383-392 }\end{array}$ \\
\hline
\end{tabular}

the role of red blood cells (RBCs) in hemostasis. This short narrative review aims to outline the available research, past and current, that reveals the role of RBCs in hemostasis, in particular blood clotting. Although early researchers suggested that RBCs were involved in this process, they had insufficient evidence to support such claims. As a result, this research received little attention from other scientists. Early researchers primarily utilized quantitative measures of RBCs, namely hematocrit or RBC count, as higher numbers of RBCs modulate blood rheology by increasing viscosity. Recent research has instead shed light on different measures of RBC function, such as expression of phosphatidylserine and adhesive proteins, aggregation, hemolysis, release of extracellular microvesicles, and erythrocyte volume. RBCs play a role in contraction of clots by platelets, and the resulting densely packed array of polyhedral erythrocytes forms an almost impermeable barrier, that is then essential for haemostasis and wound healing. Renewed interest in RBCs is primarily due to the clinically and experimentally established relationships between erythrocytes and hemostasis, that have suggested that RBCs may be potential targets for treatment of hemostatic disturbances.

Next in this issue is an original study by Sidelmann et al, ${ }^{5}$ on the abuse of anabolic-androgenic steroids (AAS), and suspected role in increasing the risk of cardiovascular disease (CVD) and cardiovascular mortality in otherwise healthy individuals. AAS abuse may increase the incidence of CVD by altering the hemostatic balance toward a procoagulant state. Studies on the effect of AAS abuse on the fibrinolytic system, however, have either demonstrated a profibrinolytic effect or no effect, though the overall effect of these compounds on fibrinolysis has not been so extensively addressed yet. The present cross-sectional study investigated the effect of AAS on fibrin clot lysis, fibrin structure, and the hemo- static proteins potentially affecting these measures in current and former AAS abusers and healthy age-matched controls. The study population consisted of 37 current and 33 former AAS abusers, along with 30 healthy age-matched controls. Fibrin clot lysis, fibrin structure properties, fibrinogen, coagulation factor XIII (FXIII) plasminogen, plasmin inhibitor, plasminogen activator inhibitor 1 (PAI-1), and thrombin activatable fibrinolysis inhibitor (TAFI) were determined. Fibrin clot lysis was significantly reduced in participants abusing AAS compared with former abusers and controls $(p<0.001)$. Plasma fibrinogen, plasminogen, and plasmin inhibitor were significantly increased in current abusers $(p<0.05)$. No significant differences were observed with respect to measures of fibrin structure properties, PAI1 , and TAFI $(p>0.05)$. In conclusion, AAS abuse seems to be associated with reduced fibrin clot lysis. This effect is not only dependent on alterations in fibrin structure but is probably caused by increased plasma concentrations of fibrinogen, FXIII, and plasmin inhibitor. Taken together, these findings would pinpoint that AAS abuse may be associated with an increased risk of developing thrombotic diseases.

Next, Aibar and Schulman provide a narrative review on new-onset atrial fibrillation (AF) in sepsis. ${ }^{6}$ Since AF is a frequently identified arrhythmia during the course of sepsis, the aim of this narrative review is to assess the characteristics of patients with new-onset AF related to sepsis and the risk of stroke and death, to understand if there is a need for anticoagulation. The authors searched for studies on AF and sepsis on PubMed, the Cochrane database, and Web of Science, finally including 17 studies. The mean incidence of new-onset AF in patients with sepsis was $20.6 \%$ (14.7\% in retrospective and $31.6 \%$ in prospective studies, respectively). Risk factors for new-onset AF included advanced age, white 
race, male sex, obesity, history of cardiopulmonary disease, heart or respiratory failure, and higher disease severity score. In-hospital mortality was higher in patients with than in those without new-onset AF in 10 studies. In four studies, the overall intensive care unit and hospital mortality rates were comparable between patients with and without new-onset AF, while three other studies did not provide mortality data. One study reported on the in-hospital incidence of stroke, which was 2.6 versus $0.69 \%$ in patients with or without newonset AF, respectively. Seven studies provided follow-up data after discharge. In three studies new-onset AF was associated with excess mortality at 28 days, 1 year and 5 years after discharge (34, 21 and 3\%, respectively). In two studies the mortality rate was comparable in patients with and without new-onset AF. Post-discharge stroke was reported in five studies, whereof two studies had no events after 30 and 90 days, one study showed a nonsignificant increase in stroke, and two studies demonstrated significantly increased risk of stroke after new-onset AF (0.6-1.6\%). The authors conclude that large prospective studies are needed to better understand the need for anticoagulation after new-onset AF in sepsis.

The issue changes focus with the next three contributions, all being related to hemophilia. First, Di Minno and colleagues discuss the enhanced half-life recombinant factor VIII concentrates (EHL-rFVIII) for hemophilia A, with insights from pivotal and extension studies. ${ }^{7}$ The development of EHL-rFVIII concentrates has improved the management of hemophilia. Furthermore, the chance of maintaining higher trough levels has allowed higher protection from bleeding and, in turn, improved safety performance for certain types of physical activity. The first technology used to improve the pharmacokinetic profile of FVIII was fusion with the Fc domain of IgG. More recently, conjugation to hydrophilic polymers of polyethylene glycol (PEG) has been demonstrated to prolong plasma half-life of FVIII by reducing the clearance of the molecule, because of steric hindrance by PEG covering the protein. The authors report the results of a systematic review of pivotal studies on EHL-rFVIII concentrates, highlighting a significant heterogeneity among different studies on EHL-rFVIII concentrates, and therefore advising that direct comparisons should be avoided. The annualized bleeding rate has ranged between 1.2 and 1.9 in different EHL-rFVIII concentrates, with progressive further decrease during extension phases of pivotal studies. Zero bleeding was reported by 40 to $45 \%$ of patients. Overall, the emerging treatment options seem to be highly effective and safe, associated with decreased dosing interval to twice weekly or less, which reduces, but does not entirely eliminate, the burden of treatment. Overall, further information is needed from real-life settings to permit differentiation between EHLFVIII concentrates and for individualizing treatment.

Next, Wang and Bai discuss mechanisms of bone remodeling disorder in hemophilia. ${ }^{8}$ As readers of this journal know, hemophilia is caused by lack of antihemophilic factor(s), for example factor VIII (FVIII; hemophilia A) and factor IX (FIX; hemophilia B). Low bone mass is widely reported in epidemiological studies of hemophilia, and patients with hemophilia are at increased risk of fracture. The detailed etiology of bone homeostasis imbalance in hemophilia remains, however, rather unclear. Clinical and experimental studies show that FVIII and FIX are involved in bone remodeling. However, it is likely that antihemophilic factors affect bone biology through thrombin pathways rather than via their own intrinsic properties. In addition, there are pathophysiological processes in several systems that might contribute to bone loss in patients with hemophilia. This review summarizes studies on the association between hemophilia and bone remodeling and might shed light on the challenges facing the care and prevention the osteoporosis and fracture in these patients.

Also discussing hemophilia is the next contribution, by Franchini and colleagues, ${ }^{9}$ this time on ABO blood group and inhibitor risk in severe hemophilia A patients. Considering the profound influence exerted by the $A B O$ blood group system on hemostasis, mainly through the von Willebrand factor and FVIII complex, the authors conducted a study evaluating the possible role of blood type on the risk of inhibitor development in hemophilia A. A total of 287 consecutive Caucasian patients with severe hemophilia A (202 without FVIII inhibitors and 85 with FVIII inhibitors), followed at seven Italian Hemophilia Treatment Centers belonging to the Italian Association of Hemophilia Centers, were included in the study. A higher prevalence of $O$ blood group was detected in patients without inhibitors as compared with inhibitor patients (55 vs. 30.6\%, $p<0.001$ ). Among the other variables analyzed (age, F8 mutation, type and intensity of treatment and treatment regimen), F8 mutation class (high-risk vs. low-risk) and treatment regimen (on-demand vs. prophylaxis) were significantly correlated with inhibitor development. However, only the effects of $F 8$ mutation and $\mathrm{ABO}$ blood type were independent of other covariates in multivariate analysis, being that non-O blood types are associated with 2.89-fold increased risk of inhibitor development. In conclusion, their study supports the protective effect of $O$ blood type on inhibitor risk in severely affected hemophilia A patients.

We move this issue of STH away from hemophilia, but continue the "bleeding" theme, with another original article, this time from Chang and colleagues, ${ }^{10}$ on the outcome of major hemorrhage at a major cardiothoracic center in patients with activated major hemorrhage protocol versus nonactivated protocol. Major hemorrhage was defined in patients ( $>16$ years) as those who received $>5$ units of RBCs in $<4$ hour, or $>10$ units in 24 hours from January 2016 to December 2018. Data was collected retrospectively from patient electronic records and Hospital transfusion databases recording issue of blood products. Of 134 patients with major hemorrhage, 24 had activated major hemorrhage protocol and 110 did not. Groups were similar for age, sex, baseline hemoglobin, platelet count, coagulation screen, and renal function, with no difference in baseline clinical characteristics. The total number of red cell units (median and [IQR]) transfused did not differ in the patients with activated (7.5 [5-11.75]) versus nonactivated (9 [6-12]) major hemorrhage protocol $(p=0.35)$. Patients in the nonactivated major hemorrhage protocol group received significantly 
higher number of platelet units (median 3 vs. $2 ; p=0.014$ ), plasma (median 4.5 vs. $1.5 ; p=0.0007$ ), and cryoprecipitate (median 2 vs. $1 ; p=0.008$ ). However, activation of major hemorrhage protocol was associated with higher mortality at 24 hours compared with patients with nonactivation of major hemorrhage protocol (33.3 vs. $10.9 \%, p=0.005$ ) and 30 -days ( 58.3 vs. $30.9 \%, p=0.01$ ). The total RBC and platelet (but not FFP) units received were higher in deceased patients than survivors. Increased mortality was associated with higher RBC:FFP ratio. Only $26 \%$ of patients received tranexamic acid, and these patients had higher mortality at 30 days but not at 24 hours. Deceased patients at 30 days had higher levels of fibrinogen than those who survived (median 2.4 vs. $1.8, p=0.01)$.

The final full-length paper in this issue of the journal is by Kvernberg and colleagues, ${ }^{11}$ and is a systematic review on platelet function and turnover in essential thrombocythemia (ET). ET is a myeloproliferative neoplasm characterized by increased platelet counts. ET has an incidence of 0.6 to 2.5 per 100,000 per year in Europe and North America. The disease is characterized by enhanced thromboembolic risk, possibly caused by increased platelet counts. Furthermore, augmented platelet function and/or increased platelet turnover may play a role. The authors aimed to explore: (1) whether platelet function and platelet turnover are increased in ET patients compared with healthy controls, and (2) whether these parameters may be associated with increased thromboembolic risk and, therefore, may support decision-making on treatment. The authors performed a systematic literature search up to March 20th, 2020 in Embase and PubMed, according to the Preferred Reporting Items for Systematic and Meta-Analysis (PRISMA) guidelines. In total, 1,923 articles were identified, 38 of which were included according to prespecified inclusion and exclusion criteria. Among the 38 studies, platelet activation (CD36 and CD62P) was investigated in 18 studies and was found to be increased in 12 of these. Platelet aggregation was investigated in 21 studies and was reported to be reduced in 20 of them. Platelet turnover (immature platelet count and mean platelet volume) was investigated in five studies, with inconclusive results. No parameters were reported to predict the risk of thromboembolic events. In conclusion, platelet activation was increased in ET patients, but platelet aggregation was reduced. The authors conclude that future studies exploring markers of thromboembolic risk in ET patients may be warranted.

As usual for these nonthematic issues of STH, we present here also some correspondence. First, Lippi and colleagues provide a brief update on periodontal disease and venous thromboembolism, where the current scientific evidencealthough limited both in time and quantity-would support the existence of a causal relationship between periodontal disease (especially severe) and venous thromboembolism. ${ }^{12}$ Second, Dall'Ara et al report an interesting case of early resolution of Heyde syndrome following transcatheter aortic valve replacement. ${ }^{13}$ Next, Dr. Krauskopf provides an exploration of the contribution of Seminars in Thrombosis and Hemostasis beyond the academic community, and using the field of "Altmetrics" (or alternative metrics). ${ }^{14}$ In brief, the main objectives of this review of altmetric data was to establish how articles otherwise highlighted in an earlier editorial ${ }^{15}$ have additionally contributed in impact by outreaching a wider audience, beyond academia. This entry is pertinent here, given the next Editorial related to the 2019 impact factor, and most highly cited papers, also appears in this issue of the journal. ${ }^{16}$ Finally, this issue of the journal concludes with a comment ${ }^{17}$ and response ${ }^{18}$ to a prior publication ${ }^{19}$ in Seminars in Thrombosis and Hemostasis, as related to use of artificial intelligence to manage thrombosis research, diagnosis, and clinical management.

We again thank all the authors to this latest issue of "Editorial Compilations" for their original and comprehensive contributions, and we hope our readership enjoys this new installment in this series.

Conflict of Interest

None declared.

\section{References}

1 Favaloro EJ. 2019 Eberhard F. Mammen Award Announcements: Part II-Young Investigator Awards. Semin Thromb Hemost 2020; 46(02):105-113

2 Danese E, Montagnana M, Gelati M, Lippi G. The role of epigenetics in the regulation of hemostatic balance. Semin Thromb Hemost 2021;47(01):53-62

3 He S, Cao H, Thålin C, Svensson J, Blombäck M, Wallén H. The clotting trigger is an important determinant for the coagulation pathway in vivo or in vitro-inference from data review. Semin Thromb Hemost 2021;47(01):63-73

4 Alamin AA. The role of red blood cells in haemostasis. Semin Thromb Hemost 2021;47(01):26-31

5 Sidelmann JJ, Gram JB, Rasmussen JJ, Kistorp C. Anabolic androgenic steroid abuse impairs fibrin clot lysis. Semin Thromb Hemost 2021;47(01):11-17

6 Aibar J, Schulman S. New-onset atrial fibrillation in sepsis: a narrative review. Semin Thromb Hemost 2021;47(01):18-25

7 Di Minno MND, Di Minno A, Calcaterra I, Cimino E, Dell'Aquila F, Franchini M. Enhanced half-life recombinant factor VIII concentrates for hemophilia A: insights from pivotal and extension studies. Semin Thromb Hemost 2021;47(01):32-42

8 Wang $\mathrm{H}$, Bai X. Mechanisms of bone remodeling disorder in hemophilia. Semin Thromb Hemost 2021;47(01):43-52

9 Franchini M, Coppola A, Santoro S, et al. ABO blood group and inhibitor risk in severe hemophilia A patients: a study from the Italian Association of Hemophilia Centers. Semin Thromb Hemost 2021;47(01):84-89

10 Chang K, Owen S, Gaspar M, Laffan M, Arachchillage DRJ. Outcome of major haemorrhage at a major cardiothoracic centre in patients with activated major haemorrhage protocol vs non-activated protocol. Semin Thromb Hemost 2021;47(01):74-83

11 Kvernberg J, Grove EL, Ommen HB, Hvas A-M. Platelet function and turnover in essential thrombocythemia: a systematic review. Semin Thromb Hemost 2021;47(01):90-101

12 Lippi G, Nocini R, Favaloro EJ. Periodontal disease and venous thromboembolism. Semin Thromb Hemost 2021;47(01): $110-111$

13 Dall'Ara G, Grotti S, Conficoni E, et al. Early resolution of Heyde syndrome following transcatheter aortic valve replacement. Semin Thromb Hemost 2021;47(01):102-104

14 Krauskopf E. The contribution of Seminars in Thrombosis and Hemostasis beyond the academic community. Semin Thromb Hemost 2021;47(01):105-109 
15 Favaloro EJ. Welcome to Seminars in Thrombosis and Hemostasis 2020-new (2018) impact factor and most highly cited papers. Semin Thromb Hemost 2020;46(01):1-5

16 Favaloro EJ. Welcome to Seminars in Thrombosis and Hemostasis 2021-new (2019) impact factor and most highly cited papers. Semin Thromb Hemost 2021;47(01):6-10

17 Martins TD, Filho RM, Romano AVC, Annichino-Bizzacchi JM. Comment and update on: "Using Artificial Intelligence to Manage
Thrombosis Research, Diagnosis, and Clinical Management. Semin Thromb Hemost 2021;47(01):112-114

18 Mishra A, Ashraf MZ. Response to "Comment and Update on: "using artificial intelligence to manage thrombosis research, diagnosis, and clinical management. Semin Thromb Hemost 2021;47(01):115-120

19 Mishra A, Ashraf MZ. Using artificial intelligence to manage thrombosis research, diagnosis, and clinical management. Semin Thromb Hemost 2020;46(04):410-418 